\title{
Turn-taking in Conversations
}

\author{
by John M. Wiemann and Mark L. Knapp
}

\begin{abstract}
The mechanisms by which people
take turns speaking in a

conversation are both spoken and

nonverbal, open and subconscious.
\end{abstract}

$A$ child of five enters a room where his mother and another woman are talking. The child tugs on his mother's skirt for attention and, without waiting for her to respond, he begins talking to her. The mother becomes irritated and scolds the child for interrupting while she is talking.

Five college students are sitting in a dorm room talking. One of the five students has been talking for about ten minutes when another member of the group says, "Jim, why don't you shut up! I can't get a word in edgewise."

In each of the preceding stories, the central figure is guilty of violating a communicative norm in our culture. And, in each case, the response was in the form of a reprimand. At least two explanations account for the reprimands given to the central figures in the stories: (a) they did not provide for a smooth transition of the speaking turn from one person to the next, and (b) they forced a definition of the situation that the other interactants present were not willing to accept. The nature of this conversational "turntaking" or "floor apportionment" will be the focus of this essay.

The phenomenon by which one interactant stops talking and another starts in a smooth, synchronized manner is considered the most salient feature of face-to-face conversation by some researchers $(12,19,20)$. The fact that we usually make judgments about people based on the way they interact argues that the structure of a conversation-the way it "comes off"-is at least as important as the content.

John M. Wiemann is a Ph.D. candidate in the Department of Communication and Mark L. Knapp is an Associate Professor of Communication, Purdue University. 


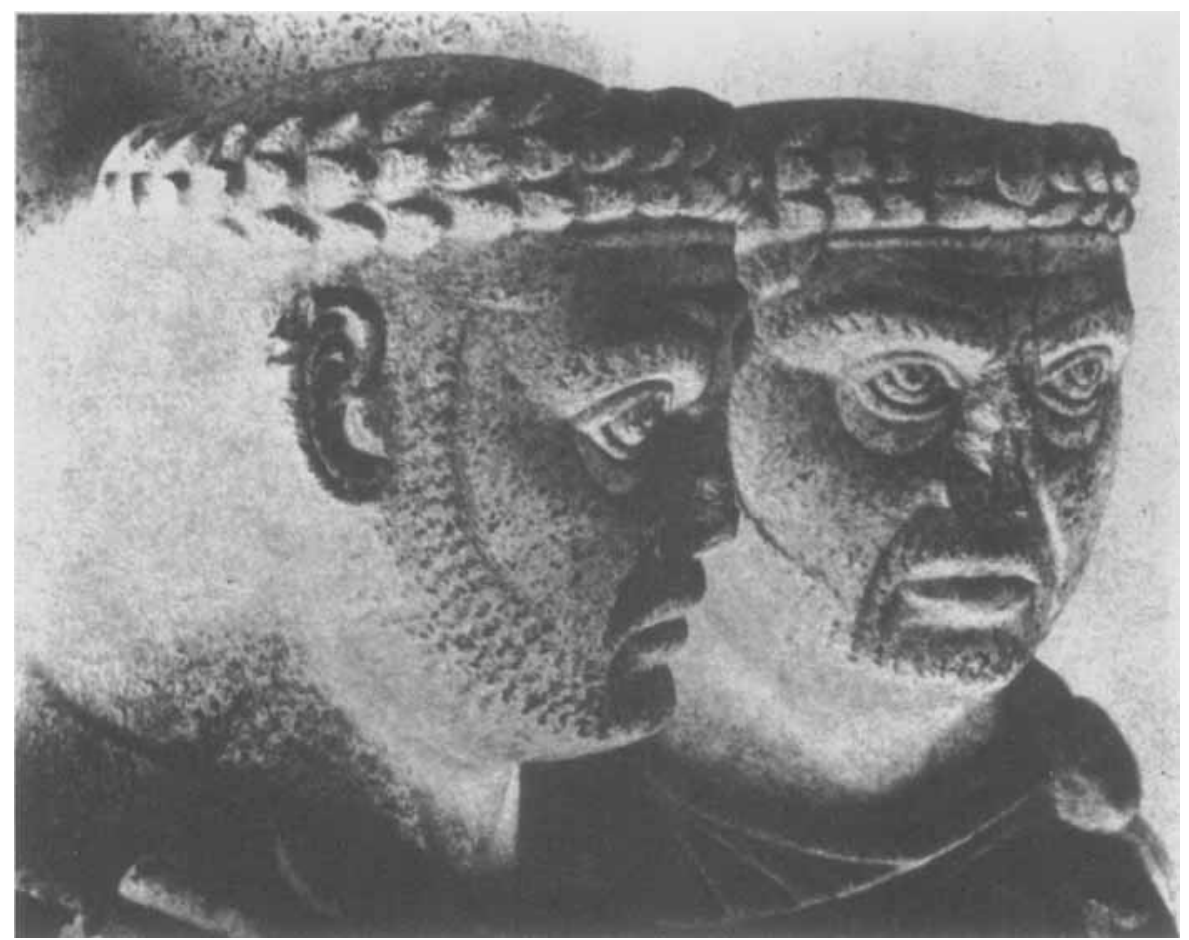

Goffman (10) recognized that when two or more people come together to interact, they are making a symbolic commitment to one another to respect the role that each chooses to play. One role that is basic to almost all other roles that an interactant can present is the role of human being, one worthy and deserving of consideration and respect.

In order to help insure that one receives the respect that each person considers his due, most cultures have developed rather elaborate (if unelaborated) rules ${ }^{1}$ to govern what should and should not be said and done in interactions. From this perspective, Goffman defines a rule as a "guide for action, recommended not because it is pleasant, cheap, or effective, but because it is suitable or just" (11, p. 48).

Unlike other societal rules (e.g., criminal laws), interaction rules are seldom specified, and consequently the actions they govern are usually carried out unthinkingly. For the most part, it is only when a rule is broken that the interactants become aware that something is amiss with the interaction, and attention is then usually directed away from the content of the conversation and toward putting the interaction back on the right track (11). Thus, in the earlier examples, the interactants interrupted their partners to remind the offenders of two existing rules: (a) one person speaks at a time, and (b) speaker-changes should reoccur (20). One result of the sanctioning action might have been embarrassment for all present-a culturally undesirable state of affairs and a rule violation in its own right.

1 The term "rules" is used here in the descriptive, rather than prescriptive, sense. 


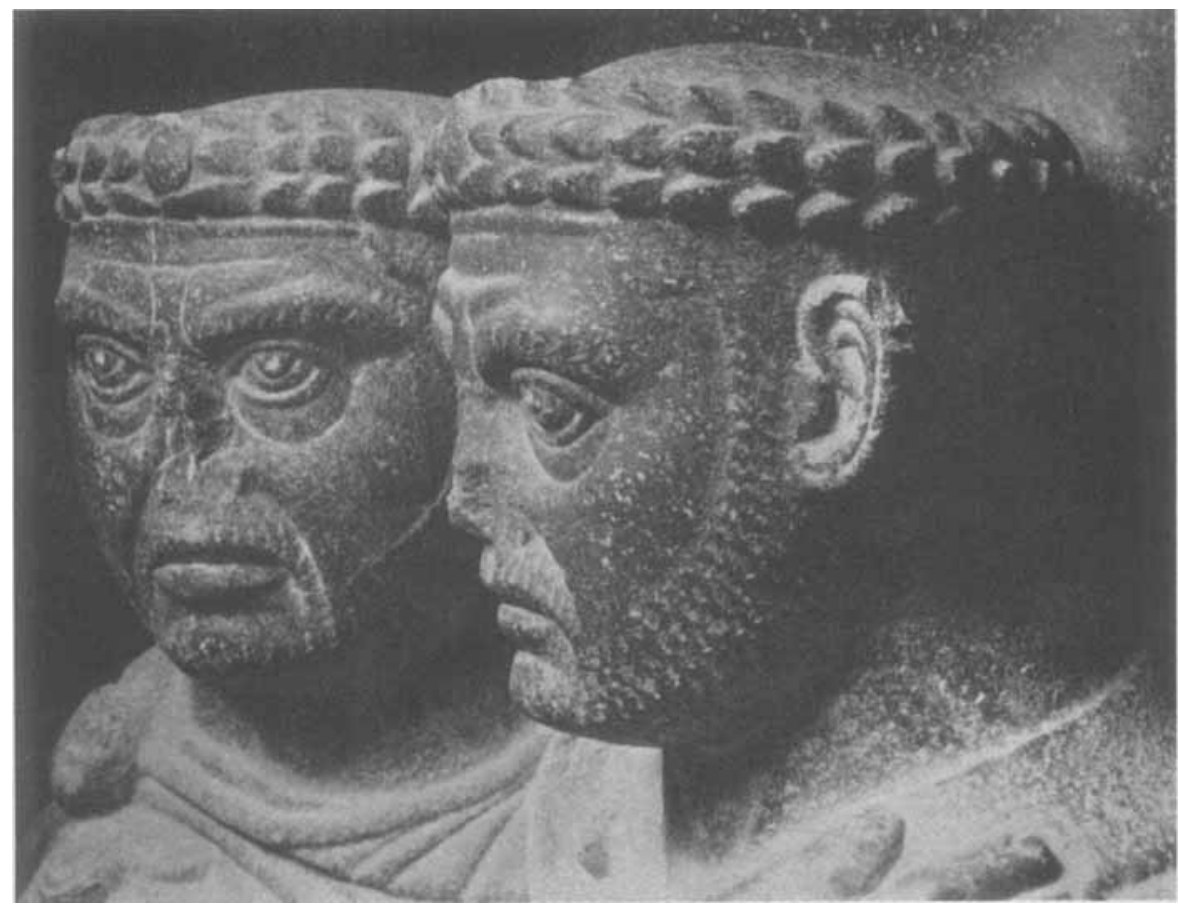

This leads to an important aspect of rule-governed interaction behavior: the manner in which specific rules are employed provides us with information about the nature of the relationship between the interactants. This seems to underline the importance of interaction rules: they directly impinge on each interactant's presented self. For example, interruptions or inattention may convey disrespect and "must be avoided unless the implied disrespect is an accepted part of the relationship" (11, p. 36).

Interaction rules can be considered from the perspective of communication theory. Communication consists of manipulating symbols; if these symbols are to be understood as intended, rules must exist for their encoding and decoding. Cushman and Whiting differentiate between two types of rules that are necessary if an interaction is to come off successfully: "(1) those which specify the action's content (its meaning, what it is to count as) and (2) those which specify the procedures appropriate to carrying out the action" (14, p. 217).

Included in Cushman and Whiting's procedural rules category are the interaction rules that have been discussed above. When a rule for symbol by which a rule is implemented) has gained wide acceptance by a particular culture, it can be said that that rule (symbol) has achieved "standardized usage." In other words, certain situations or symbols have particular meanings and require particular responses. If an individual fails to respond correctly to a situation or symbol (because the individual either does not know the appropriate rules to govern his response or does not wish to 
respond in the appropriate fashion), that person is alienating him or herself from those present. ${ }^{2}$

The way interaction is regulated in an elementary school classroom is an example of what we have been discussing. When children enter first grade, they are often told they must raise their hands before speaking. The rule is: "You may not speak unless the teacher gives you permission." The symbol by which the rule is implemented-by which permission is requested and gained-is the upraised hand. New students may be given some amount of time in which to learn the rule. Note that learning the rule and learning the symbol to implement the rule are, for all practical purposes, one and the same thing. During the learning period, violations of the rule are corrected, but tolerated. The assumption is that the children are not yet part of the school-culture. After a certain period, the teacher decides that all of the first graders have had enough time to become acculturated - that is, they have had enough time to learn the rules. Now when the hand-raising rule is violated, the children are likely to be punished; disrespect and disregard for the school-culture is assumed.

This rather ordinary example illustrates that people often rely on conformity to interaction rules-particularly turn-taking rules-for information about an individual's relationship with or orientation towards a group or individual. Speier states this same point more strongly:

Cultural competence in using conversational procedures in social interaction not only displays adequate social membership among participants in the culture, but more deeply, it provides a procedural basis for the ongoing organization of that culture when members confront and deal with one another daily (19, p. 398, emphasis in original).

The conversational procedures Speier is concerned with are the turn-taking phenomena.

In order to communicate successfully, an interactant must understand and subscribe to the interaction rules of his culture. The violation of interaction rules provides us with information about an individual's orientation toward his or her fellow interactants.

\section{Our specific topic is the process of deciding who will speak and who will listen in an interaction.}

With the exception of people in classrooms or those attending formal meetings, individuals usually devote little conscious time to deciding who will speak. In our culture we have no formal system for determining who will speak at "informal" gatherings. In other words, there is no cultural norm that states, for example, that when adults are sitting around the dinner table, the person sitting on the north side of the table will speak

${ }^{2}$ Goffman (11, pp. 113-136) treats this type of alienation in great detail. 
first and thereafter the speaking turn will rotate in a clockwise direction. ${ }^{3}$

Yet we often evaluate our interactions in terms of the allocation of speaking roles. A person who dominates a conversation is often judged a "boor" (particularly if the "judge"-the other interactant-had something to say and did not get the opportunity). The person who constantly interrupts is judged "rude." Thus, it may be embarrassing and rude to tell the boor to give someone else a chance to speak.

In spite of this seeming paradox, there are numerous encounters in which the need to interrupt someone else to get the floor does not confront us. If interactants need not resort to violence-either verbal or physical-to get the floor, how do they decide who will speak and who will listen? In other words, how are the numerous interaction rules governing the respect of the speaker implemented?

The behavior by which an exchange of speaking turns is accomplished will be referred to here as the turn-taking mechanism. While a number of studies have dealt with various behaviors which may be part of the turntaking mechanism, only Duncan $(6,7,8)$ has dealt directly with it in its entirety. Taking an inductive approach, Duncan observed interactions, and then described the behavior that accompanied speaking-role changes. ${ }^{4}$

Duncan $(7,8)$ posits three rules operating during successful exchanges of the speaking turn: (1) turn-yielding cues, which are used by speakers to signal that they are concluding their remarks and that the auditors can take the floor; (2) suppression of speaking-turn claims, which are exhibited by speakers in conjunction with turn-yielding cues, when they intend to maintain their speaking turn; and (3) back channel cues, which are displayed by the auditors to indicate that they do not wish the floor, even though the speaker is displaying yielding cues.

Turn-yielding. The rule for turn-yielding states that the auditor may take his speaking turn when the speaker emits any one of a number of verbal or nonverbal turn-yielding cues. The display of a turn-yielding cue does not require the auditor to take the floor; he may remain silent or reinforce the speaker with a back channel cue. The absence of simultaneous turns (i.e., both participants in the conversation claiming the speaking turn at the same time) during the exchange of speaking roles is considered a successful exchange. If the turn-taking mechanism is operating properly, the auditor will take his turn in response to a turn-yielding cue emitted by the speaker, and the speaker will immediately yield his turn.

Duncan (8) defines a turn-yielding signal as the display of at least one

3 This is not the case in all cultures, however. Albert (1), for example, reports that the Barundi of Africa do have such a formal system. When in a conversational gathering, the person from the highest caste (or status) will speak first, followed by the person from the second highest caste, etc., until everyone present has had a speaking turn. Then the interactants repeat this sequence of speakers until the interaction is terminated.

4 The data consist of the first 19 minutes of two dyadic conversations, one between a therapist and his patient and the other between the same therapist and one of his colleagues. 


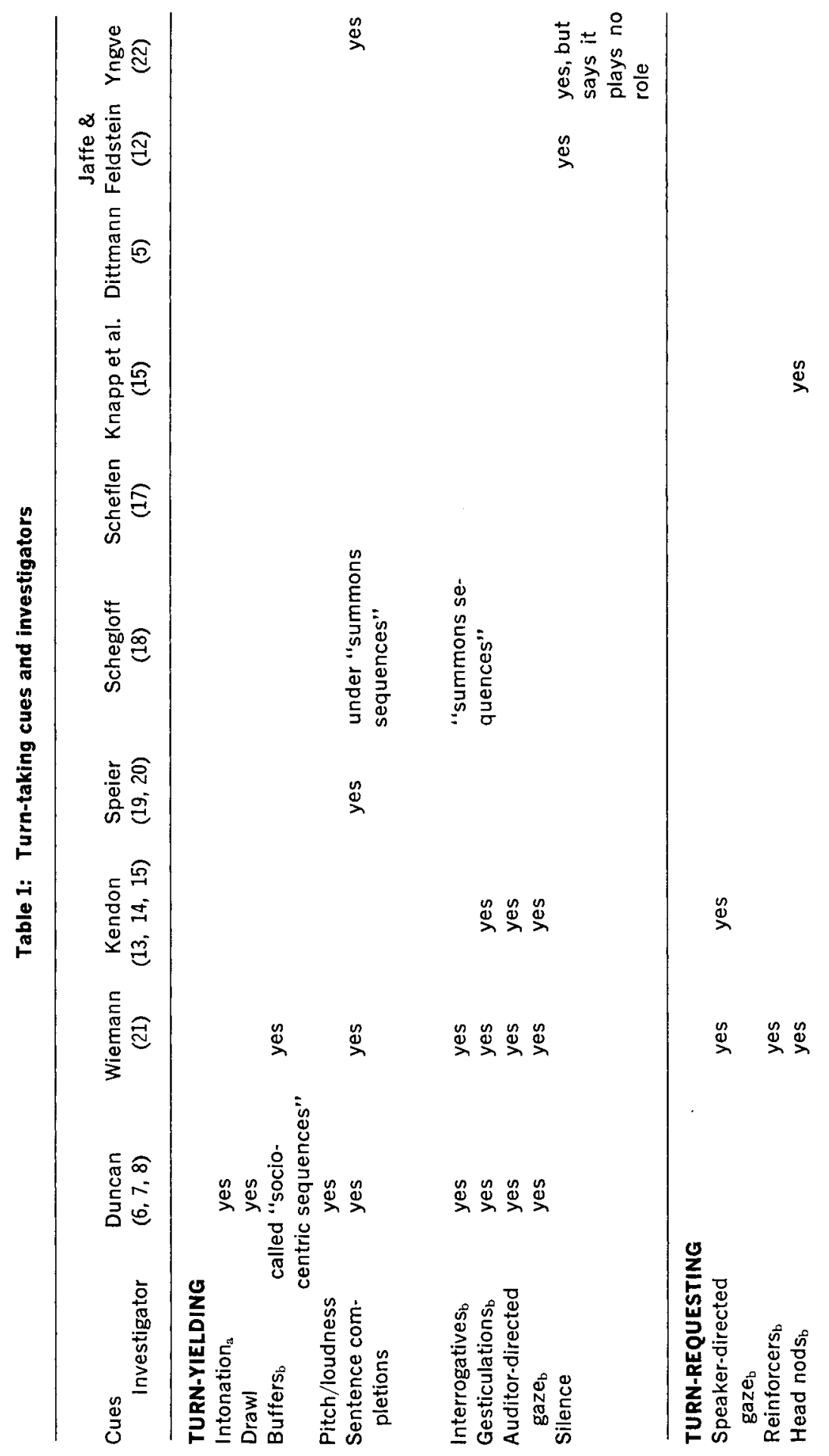




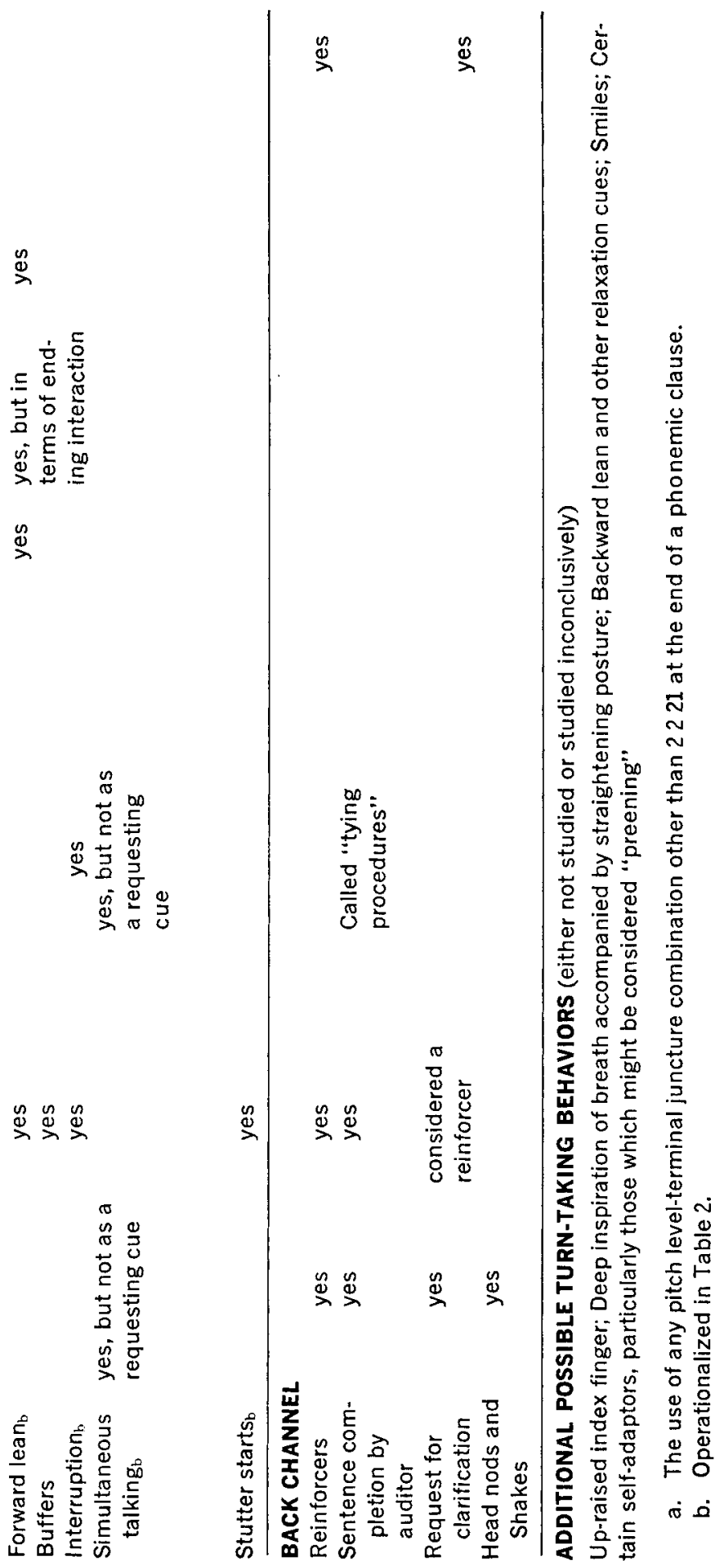


of a set of cues (see Table 1) either singly or in clusters. He notes that, while some of the cues may be displayed at any time during the conversation, they are only considered part of the turn-taking mechanism if they occur at the end of a phonemic clause. Others have found, however, that the change in frequency of cues over a speaking turn or the intensity of cue display may be the determining factor in how a behavior is interpreted by the other interactant (cf. 21).

Suppression of speaking-turn claims. This cue serves to maintain the turn for the speaker by counteracting any turn-yielding cues emitted simultaneously. It consists of "one or both of the speaker's hands being engaged in gesticulation" (8, p. 38). Self- and object-adaptors ${ }^{5}$ do not function as claim suppressors.

Back channel cues. These cues, exhibited by the auditor, are related to various speaker signals, either within-turn signals (8) or turn-yielding cues. In relation to turn-yielding cues, they serve to signal the speaker that the auditor does not wish to take the speaking role (cf. 22).

Turn-requesting. Personal experience tells us that participants in a conversation are not at the mercy of the speaker, in spite of the interaction rules. To account for this, Wiemann (21) proposed that a turn-requesting rule exists, and that auditors employ such a rule to let the speaker know that they want the floor without violating the respect due the speaker because he or she is the speaker.

Turn-requesting consists of the display of one or more of a number of verbal or nonverbal cues (cf. Table l) by the auditor. If the turn-taking mechanism is functioning correctly, the speaker should relinquish the speaking role upon completion of the thought unit he or she is communicating at the time the request is made.

Wiemann (21) took a more deductive approach than Duncan in studying the floor yielding and requesting aspects of the turn-taking mechanism. In light of the concept of "standardized usage" mentioned earlier, he asked if (in a given homogeneous group of subjects) certain verbal and nonverbal behaviors would manifest themselves in such a way that they might be interpreted to be operating as turn-taking cues.

Subjects were 18 Purdue University students, each paired with an acquaintance such that there were three all-male dyads, three all-female dyads, and three dyads composed of one male and one female.

Dyads were videotaped while they discussed a topic suggested by them and approved by the researcher. A random sample of 72 interaction sequences or exchanges was drawn from the tapes and analyzed twice, once for each subject as a speaker and once for each subject as the auditor.

A review of literature, observation in a number of different settings, and introspection led to the construction of two analysis systems, one for verbal behavior and one for nonverbal behavior (cf. Table 2).

\footnotetext{
5 Ekman and Friesen (9) discuss self-and object-adaptors in detail. Generally, adaptors consist of manipulation of either the self (i.e., scratching) or personal objects (e.g. re. arranging one's clothes or handling a pipe).
} 
Table 2: Behavioral analysis categories for the turn-taking mechanism

Nonverbal
time spent looking at the facial area
around the eyes of the other person.

2. Smiles. A positive facial expression marked by upturned corners of the mouth (as opposed to a straight or down. turned mouth)

3. Reclining angle. When that plane defined by a line from the communicator's shoulders to his hips is away from the vertical plane, such that the communicator is leaning backward to some degree.

4. Forward-leaning angle. When that plane defined by a line from the communicator's shoulders to his hips is away from the vertical plane, such that the communicator is bending forward at the waist.

5. Gesticulations. Hand and arm movements (excluding self-manipulations), including side-to-side, forward-back, and up-and-down movements (e.g., an upraised and pointed index finger).

6. Head nods. Cyclical up-and-down movements of the head.

\section{Verbal}

1. Interrogative request. A question specifically directed to the other dyad member.

2. Completion. The completion of a declarative "statement" with no attempt being made by the speaker to continue.

3. Buffers. Short words or phrases which are "content-free," more or less stereotypical, and which either precede or follow substantive statements (e.g., "but uh," "you know," "or something," "um," "well," and "uh-well").

4. Interruption. The attempt to assume the speaking role before it has been relinquished by the current speaker.

5. Simultaneous talking. Speaking by both interactants at the same time. (This includes simultaneous turns, where both speakers attempt to hold the floor at the same time.)

6. Stutter starts. Short words (including nonfluencies) or phrases repeated with increasing frequency by one interactant while the other interactant holds the speaking role (e.g., "I. . . I. . . I. . . I think we should vote now.")

7. Reinforcers. Words that provide feedback to the speaker, but do not neces. sarily attempt to gain the speaking role for the interactant emitting them. Short questions asking for clarification are coded in this category. (Examples include: "Yeah," "yes," and "um-hm.")

The verbal behaviors that played a role in turn-yielding were (in order of incidence): completions, interrogative requests, and buffers.

The only nonverbal behavior that seemed to play a role in turn-yielding was other-directed gaze. An analysis of the time spent in other-directed gazes indicated that speakers steadily increased the amount of time spent looking at the auditor as their speaking turn approached completion. This phenomenon was also noted by Kendon (13). Other nonverbal behaviors observed, including the termination gestures specified by Duncan (8), seemed to play little or no role in the turn-yielding of these subjects. 
Verbal turn-requesting behavior included (again, in order of occurrence): simultaneous talking, buffers, reinforcers, interrogative requests, and stutter starts.

Nonverbal turn-requesting behavior included other-directed gazes and head nods. While the other nonverbal behaviors included in the study were emitted by the auditors, they seemed to play little or no role in turnrequesting for these subjects.

In the Wiemann study, the two verbal behaviors that seemed to play a significant role in the turn-yielding mechanism were completions and interrogative requests. Both of these behaviors can be considered "natural" endings to utterances; the speaker is allowed to complete the utterance he or she is engaged in before the other interactant assumes the floor. In fact, the speaker was allowed to come to a "natural" conclusion in 67 of the 72 interaction episodes studied-93 percent of the time.

There is little need to discuss the effectiveness of the interrogative "statement" in causing a change in the speaking role. One of our cultural norms seems to be that we answer questions that are asked of us (18).

The tendency to effect a change in the speaking turn by ending an utterance with a declarative "statement" is not as easily explained. The declarative statement does not have the same "demand" characteristics as either an interrogative or an imperative statement. Schegloff (18), however, contends that certain declaratives-which he calls summonses--do possess the same demand characteristics as questions.

One possible explanation for the high incidence of "completion" endings is that the silence of the speaker at the end of an utterance (failure to continue speaking) operates as a yielding strategy. Jaffe and Feldstein (12) have systematically investigated silence or length of pauses as part of the turn-taking mechanism. They report that the longer the silence during a conversation, the more likely it is that a change in speaker will take place. Further, they found that pacing depends on silences and that "the pacing of conversational interaction is (a) characteristic of the speakers involved, (b) stable within any conversation, and (c) consistent from one conversation to the next for the same two speakers" (p. 116).

Thus, it seems probable that two acquaintances would develop a rhythm of exchange of turns; because of their experiences interacting with each other, they would learn each other's style and could "predict" when each would stop talking. Jaffe and Feldstein's findings are not supported by Yngve (22), who in a preliminary report of his research on turn-taking states that silence alone does not play a major role in speaker switches.

Buffers apparently had little to do with turn-yielding for Wiemann's informed dyads. Speakers seemed to emit buffers in attempts either to hold the floor while planning their next statements or to elicit feedback from the auditor without yielding the Hoor (i.e., eliciting head nods or reinforcers for continuing to talk). "She's gonna wear, you know, that low-cut dress ..." is an example of this use of buffers. The interactant uttering this statement was responding to a question and continued the description of 
the wardrobe for several more seconds. The purpose of the "you know" was clearly not to yield the floor.

The fact that, in our culture, auditors pay respect to the speaker simply because he is the speaker has been discussed at length. The high frequency of "natural" completions and the use of occasional buffers by the speakers seems to indicate that speakers feel a certain responsibility to their auditors-almost as if they had a "the show must go on" attitude toward the speaking turn. This is not to deny that speakers talk for a variety of other reasons as well. They do, however, seem to feel a need to fill the silence. If this tentative proposal has some validity, it helps to explain the general lack of verbal yielding behavior. Speakers feel it is their "duty" to keep the interaction alive; if auditors want to talk, then they must let the speakers know that they are ready to assume responsibility for the interaction.

A competing explanation for the reluctance to yield the floor is the demand characteristic of the experimental situation. The students who served as subjects might have felt they had to keep the conversation going or the "experiment" would fail.

Since the nonverbal channel is often more subtle than the verbal, speakers can be expected to make more use of it. Probably the most frequently occurring nonverbal yielding behavior is auditor-directed gazes. The percentage of time spent by the speaker looking at the face of the auditor increases steadily as the speaking turn approaches finality from 61 percent in the first third of the interaction episode to 83 percent in the final third. ${ }^{6}$ Conversely, the percentage of time the auditor spends looking at the speaker increases from the first third to the second third of the interaction episode, and then drops off in the final third (see Figure 1).

As the speaker comes to the end of an utterance, he or she looks at the auditor in search of feedback. If this feedback is in the form of a verbal response, then the speaking role is likely to be exchanged. The looking away by the auditor seems to be a response to the speaker-acknowledging the turn-yielding cue and the acceptance of the speaking turn.

It seems that other-directed gazes function more as a turn-yielding device than as a turn-requesting device, but they can function as both. It is possible that there must be mutual gaze at, or very near, the point where the speaking roles are exchanged. That is, for other-directed gaze to be successful as a turn-taking strategy, the two interactants must have eye contact at or near the exchange point. The increasing percentage of time the speaker spends in auditor-directed gazes as the episode draws to an end seems to indicate that the speaker is making himself more available for that

B A review of the video tapes of subjects conversing indicated that changes in the duration of some nonverbal behaviors might present a more accurate picture of the role these behaviors play in the turn-taking mechanism than did the frequency counts orginally computed. Therefore, the duration of other-directed gazes, smiles, reclining angle and forward-leaning angle was calculated for each interaction segment (i.e., for each person during any one speaking turn). In order to make interaction segments of different lengths comparable, they were divided into thirds. Thus, what happened in the beginning of one interaction segment (in the first third) could be compared with all other segments. 
bit of eye-contact that will aid in the exchange of the speaking turn.

The role that gesticulations play in the turn-taking mechanism is not entirely clear. Duncan $(6,7,8)$ reports that his subjects used gesticulations as turn-yielding signals. On the other hand, Wiemann (21) found a very low incidence of gesticulations - too low to draw any meaningful conclusions. A number of factors may account for low incidence of gesticulations in some situations. The setting may encourage interactants to tone down their behaviors-not just gesticulations, but all overt behaviors. Also, the topic may or may not encourage excited or animated behavior. For example, conversations concerned with describing an unknown event to an auditor or one that is particularly salient to either party may encourage more animated behavior.

The role that shifts of posture play in the turn-taking mechanism is unclear. The available data-which are unconvincing-suggest that it plays no role at all. However, Kendon $(14,15)$ and our own natural-state observations argue against this interpretation. People do not really sit still in their seats for an appreciable length of time. Auditors may be reclining for a time and then, as they prepare to take the speaking role, move to an upright position or even to a forward-leaning position. Likewise, speakers at times "punctuate" their yielding of the floor by leaning back in their chairs as they finish their utterances.

Situational variables may influence the way postural shifts are used as turn-taking cues. Gross postural shifts may be unnecessarily harsh or obvious forms of conversation regulation in some situations. Interactants who know each other may find the more subtle turn-taking cues (head nods, other-directed gazes, buffers) sufficient to effect changes in the speaking turn.

Auditors seem to make more use of the verbal channel in turn-taking than do speakers. 'This is probably true, in part, because the speakers are using the verbal channel to convey the substance of their message. Some form of verbal behavior accompanied 49 percent of the exchanges of the speaking turn in the Wiemann study. That is, auditors engaged in some form of talking immediately prior to assuming the speaking turn. ${ }^{7}$

The generalized requesting cue which appeared most frequently was simultaneous talking. Because simultaneous talking can take a variety of forms (buffing, reinforcing, etc.), it can serve a variety of purposes in the conversation, not all of which have to do with the turn-taking mechanism. However, the high percentage of simultaneous talking immediately before exchanges of speaking roles indicates that people use it as a requesting strategy.

Buffers and reinforcers are frequently-used turn-requesting strategies. Buffers generally constitute a clear attempt by the auditor to get the floor.

7 A person is considered to "have the floor" when, through tacit, but mutual agreement of those participating in the interaction, he has the "right" to speak uninterrupted. This agreement is inferred from some signal by one of the other participants or by their attentive silence. An interactant's turn begins when his speech becomes audible and ends when another interactant takes over the floor. 


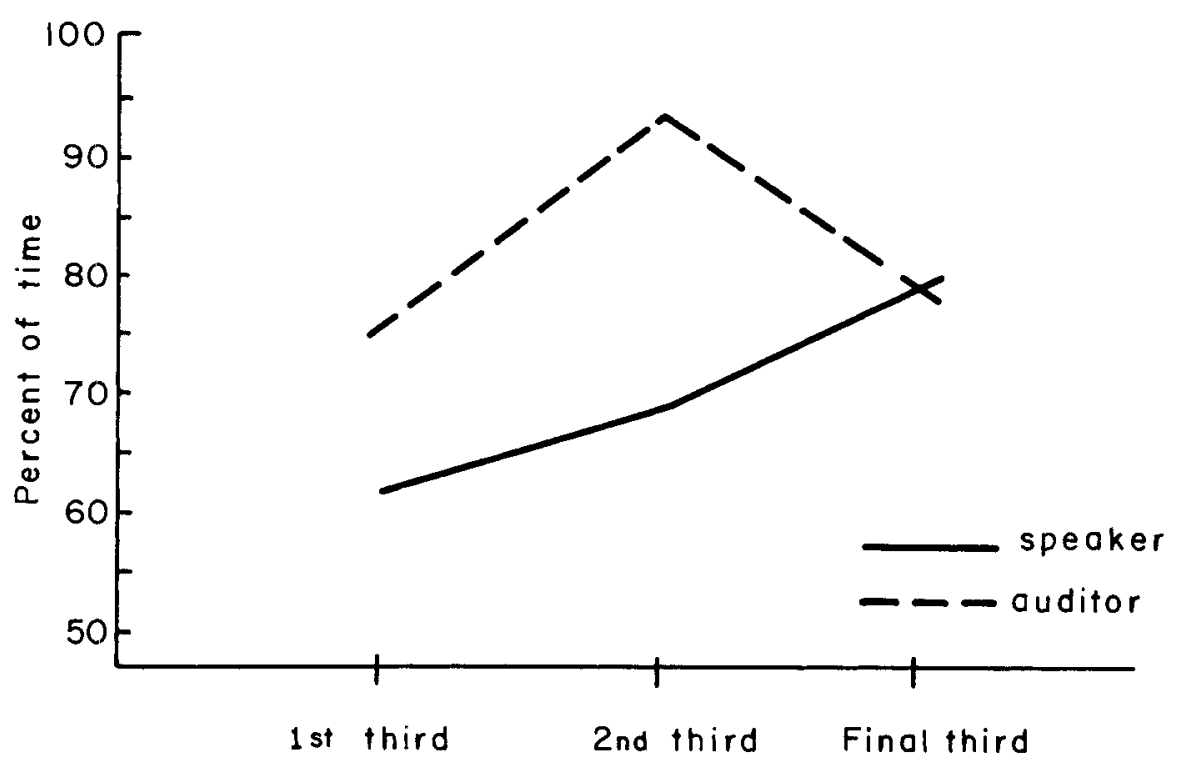

Segment of interaction

Figure 1. Percentage of time spent in other-direct gazes

Occasionally, the buffers are uttered while the speaker is talking; but more often they are uttered while the speaker is silent, either during a pause or after the speaker has clearly ended his utterance. In the latter case, they seem to be a signal by the auditor that he is ready to talk; the buffers allow the other interactant time to attend to the new speaker before he begins his "message." The use of buffers by the auditor at this point may also constitute a signal to the speaker that he has accepted the speaker's offer of the floor.

The role of reinforcers in turn-requesting differs from that of buffers. While these two phenomena are similar, reinforcers have usually been considered a form of encouragement for the speaker to continue talking (cf. $6,7,8$ ). However, the subjects in this study seemed to use reinforcers in order to effect a "request" for the floor at least as often as they used them to encourage the speaker to continue talking. Knapp et al. (16) speculate that reinforcers are used in this way when one interactant wants to get the floor or when "we interact with verbose persons, where conversational 'openings' are difficult to find." In instances such as these, the reinforcers may be used to encourage the speaker to "hurry up and get finished" so the auditor can assume the speaking role.

The behavior of the auditor that accompanies the utterance of the reinforcer, or the behavior of the speaker to which the reinforcer is a response, probably determines how the speaker interprets the reinforcer. 
If the utterance of the reinforcer is accompanied by a postural shift (e.g., a move from a reclining to an upright position), prolonged eye contact, or rapidly repeated and demonstrative head nods, the reinforcer is more likely interpreted as a request for the floor. Reinforcers also seem to have the force of a request when they are uttered while the speaker is talking (rather than during a pause) or if they are uttered while the speaker is not looking at the auditor. On the other hand, if the speaker has somehow requested feedback from the auditor (e.g., by the use of a buffer like "you know"); if the reinforcer comes during a pause by the speaker; or if it is delivered in a slow, thoughtful manner, the speaker seems to interpret it as encouragement to continue talking. It may be that a dramatic increase on an "activity dimension" differentiates between turn request and back channel cues.

\section{At times, an utterance seems to function both as a reinforcer and as a buffer.}

For example, after a speaker makes a "statement" (or during the speaker's utterance), the other interactant might use a "hybrid" reinforcer-buffer to effect a change in the speaking role. An example of this type of expression is "yeah, well." The auditor is giving the speaker a strong indication that he (the auditor) wants the floor. The use of the buffer seems to be an attempt to soften the impact of the request (particularly if it is an interruption) and to maintain the integrity of the interaction. The use of "well" or "but" might not soften the request for the floor at all. Such a requesting strategy often causes speakers to try harder not to let the requester "in" because they know disagreement is coming. It is a clear signal, but not necessarily subtle, soft, or effective.

More than any of the other verbal behaviors mentioned here, buffers and reinforcers seem to bind the interactants together. Not only do they serve as attention signals and turn-taking signals, but reinforcers and buffers also provide the auditor a means of participating in the conversation in an overt, verbal manner even when he or she doesn't have the floor. Using these two behaviors as a means of participation, the auditor does not violate the "don't interrupt the speaker" norm; and, therefore, this form of participation is acceptable in our culture.

Interrogative requests, when used by the auditor, seem to serve a function similar to those served by reinforcers. That is, interrogative requests are in the back channel and encourage the speaker to continue by asking for explanation or clarification. They are included here as part of turn-requesting behavior because, in some instances, the auditors append their answer to the question asked; thus, they allow the auditor to briefly hold the floor without actually taking the speaking turn. For example, one interactant described to his partner a Purdue basketball game he had seen the night before. He said, in part, ". . . the guards played well last night." Before he finished the utterance, the auditor said, "You mean Parkinson? He's great." The speaker continued speaking "over" the auditor's comment 
(he recognized the comment with a head nod); he did not slow down but he did go on to elaborate on Parkinson's play. While this may have been the direction the conversation would have taken anyway, it seems that the auditor's comment about Parkinson encouraged the speaker to talk about Parkinson. This interaction episode ended with an exchange of the speaking turn a few "statements" later when the speaker asked the auditor what he thought about Parkinson.

Stutter-starts appear to be similar to buffers as far as the role they play in turn-requesting. Stutter-starts may constitute more of a demand for the floor than do buffers, however. They are usually emitted by the auditor only after the speaker has had the floor for some time (15 to 20 seconds) or when the speaker pauses longer than usual. This could be because the speaker is not attending to the more subtle requesting cues of the auditor or because he inadvertently gives a turn-yielding cue and then continues to talk- "faking out" the auditor, so to speak.

The role speaker-directed gazes play in turn-taking has already been discussed. It seems worth repeating that mutual gaze is necessary if this is to serve as a turn-taking strategy.

Head nodding appears to play a major role in turn-requesting, while having little or no significance in turn-yielding. Speakers do not systematically increase the amount of nodding as the episode progresses. On the other hand, there is a dramatic increase in nodding by the auditor.

The rapidity of the head nods and whether or not they are accompanied by any verbal behavior seems to determine how speakers interpret them. Closely placed, successive nods, and nods that accompany short, rapid-fire reinforcers (e.g., "yeah, yeah") or buffers (e.g., "uh, uh"), constitute requests for the floor by the auditor. On the other hand, nods that serve as responses to a request for feedback from the speaker seem to be interpreted by the speaker as support for his maintaining the floor.

Both nodding and other-directed gazes appear to be important to the turn-taking mechanism because of the dual role they play. These behaviors indicate support for and interest in the other interactant when they are displayed by one member of the dyad. They also provide both the speaker and the auditor with a means of either yielding or requesting the floor. Their supportive nature "softens" the terms of the exchange. The nodding auditor is signaling the speaker his agreement and reinforcement at the same time the request is being made; the respect of the speaking role is maintained because, in effect, the auditor is letting the speaker know that there is no threat or disrespect intended by the takeover of the floor. In like manner, the speaker can use nodding and auditor-directed gazes to inform the auditor that he is looking for feedback and that he is receiving the auditor's messages. All this can be accomplished without interrupting the flow of the "conversation."

While primarily yielding cues, gesticulations may also be used by auditors to signal in a rather dramatic way their desire to get the floor. An example of this use of gesticulation is the pointed and raised index 
finger-usually accompanied by an open mouth, poised to speak, or other such behavior that is similar to school-trained hand-raising.

Other nonverbal cues not yet specifically studied, but whose co-occurrence with reinforcers might influence the interpretation of reinforcers, include a deep inspiration of breath immediately before the reinforcer is uttered and the holding open of the mouth for a brief period before the reinforcer is uttered (21).

As is the case with most exploratory work, the results of these investigations are more suggestive than conclusive. Some of the behaviors studied were used in the same ways consistently enough to warrant the tentative conclusion that they play a role in the turn-taking mechanism.

Many of the behaviors mentioned above are emitted simultaneously. In some instances, it appears that emission of two or more cues at the same time may determine the other interactant's interpretation of the cues. For example, a head nod by the auditor is interpreted as a back channel cue or reinforcer if displayed alone, but when the nod is accompanied by "yeah, but," it becomes a request for the floor. The appearance of multiple cues may also be related to the perceived urgency or strength of turn-yielding or turn-requesting. For example, when the auditor leans forward in his chair, raises and points his finger and says "yeah ... yeah ... yeah, yeah" while nodding his head, he is giving the speaker a very strong indication that he would like the floor.

Auditors displayed a wider variety of verbal and nonverbal behavior and display those behaviors more frequently than do speakers. A possible explanation for this was touched on earlier. Speakers may feel an obligation to keep the conversation going; it is their way of reciprocating the respect auditors show them (by not interrupting). This, in turn, might be one reason why auditors' respect is due the speaker. Any behavior by the speaker that might be interpreted as a unilateral move to end the encounter might be offensive (cf. 16). Turn-yielding, therefore, must be executed carefully. In normal conversation the bulk of the burden for initiating exchanges of the speaking turn-at least in terms of frequency and variety of cues displayed-falls to the auditors.

The line of thinking developed here suggests that a "grammar" of dyadic interaction exists. This is not to imply that there is any relationship between this grammar and that proposed for spoken languages. Rather, the term "grammar," as used here, implies the existence of a structure of interaction. By correctly applying the rules of this structure, interactants can accurately express their relationship to the other interactants without interrupting the flow of the content of the conversation.

Argyle $(2,3)$ has suggested that social competence can be studied much as motor skills are, with similar implications. If the components of "successful" interaction can be isolated, they can be analyzed and taught. Argyle lists four components of social competence: (1) perceptual sensitivity, (2) basic interaction skills, (3) rewardingness, and (4) poise (3, pp. 78-79). While 
all of these come into play even in something so elementary as negotiating the speaking turn, basic interaction skills seem to be at the heart of the matter: "To be socially competent it is necessary to be able to establish a smoothly meshing pattern of interaction with other people" (3, p. 78).

\section{A competence paradigm may provide commun- ication scholars with a theoretical framework for the study of interpersonal communication.}

Turn-taking in conversations not only helps us apportion the floor, but also serves a symbolic function of helping the interactants to define their relationship. The way in which this ritual is managed by one interactant will affect the judgments made about him or her by the other interactant. Thus, research may show that it is the management of the small, unnoticed, ritualistic behaviors that has the greatest effect upon our attributions about others; it may be these behaviors that determine whether or not we are successful interactants.

\section{REFERENCES}

1. Albert, E. M. "Cultural Patterning in Speech Behavior." In J. J. Gumperz and D. Hymes (Eds.) Directions in Sociolinguistics. New York: Holt, Rinehart \& Winston, 1972.

2. Argyle, M. Social Interaction. Chicago: Aldine Atherton, 1969.

3. Argyle, M. The Psychology of Interpersonal Behaviour (2d ed.). Baltimore: Penguin, 1972.

4. Cushman, D., and G. Whiting. "An Approach to Communication Theory: Toward Consensus on Rules." Journal of Communication 22, 1972, pp. 217-238.

5. Dittman, A. T. "Developmental Factors in Conversational Behavior." Journal of Communication 22, 1972, pp. 404-423.

6. Duncan, S. "Towards a Grammar for Floor Apportionment: A System Approach to Face-to-Face Interaction." In Proceedings of the Second Annual Environmental Design Research Association Conference. Philadelphia: Environmental Design Research Association, 1970.

7. Duncan, S. "Some Signals and Rules for Taking Speaking Turns in Conversations." Journal of Personality and Social Psychology 23, 1972, pp. 283-292.

8. Duncan, S. “Toward a Grammar for Dyadic Conversation." Semiotica 9, 1973, pp. 29-46.

9. Ekman, P., and W. V. Friesen. "The Repertoire of Nonverbal Behavior: Categories, Origins, Usage, and Coding." Semiotica 1, 1969, pp. 49-98.

10. Goffman, E. The Presentation of Self in Everyday Life. Garden City, N.Y.: Doubleday Anchor, 1959.

11. Goffman, E. Interaction Ritual. Garden City, N.Y.: Doubleday Anchor, 1967.

12. Jaffe, J., and S. Feldstein. Rhythms of Dialogue. New York: Academic Press, 1970.

13. Kendon, A. "Some Functions of Gaze-Direction in Social Interaction." Acta Psychologica 26, 1967, pp. 22-63.

14. Kendon, A. "Movement Coordination in Social Interaction: Some Examples Described." Acta Psychologica 32, 1970, pp. 101-125.

15. Kendon, A. "Some Relationships Between Body Motion and Speech: An Analysis of of an Example." In A. W. Siegman and B. Pope (Eds.) Studies in Dyadic Communication. Elmsford, N.Y.: Pergamon Press, 1972. 
16. Knapp, M. L., R. P. Hart, G. W. Friedrich, and G. M. Shulman. "The Rhetoric of Goodbye: Verbal and Nonverbal Correlates of Human Leave-Taking." Speech Monographs 40, 1973, pp. 182-198.

17. Scheflen, A. E. "The Significance of Posture in Communication Systems." Psychiatry 17, 1964, pp. 316-331.

18. Schegloff, E. A. "Sequencing in Conversational Openings." In J. J. Gumperz and D. Hymes (Eds.) Directions in Sociolinguistics. New York: Holt, Rinehart \& Winston, 1972.

19. Speier, M. How to Observe Face-to-Face Communication: A Sociological Introduction. Pacific Palisades, Calif.: Goodyear, 1973.

20. Speier, M. "Some Conversational Problems for Interactional Analysis." In D. Sudnow (Ed.) Studies in Social Interaction. New York: Free Press, 1972.

21. Wiemann, J. M. "An Exploratory Study of Turn-Taking in Conversations: Verbal and Nonverbal Behavior." Unpublished M.S. thesis, Purdue University, 1973.

22. Yngve, V. H. "On Getting a Word in Edgewise." In M. A. Campbell et al. (Eds.) Papers From the Sixth Regional Meeting, Chicago Linguistics Society. Chicago: Dept. of Linguistics, U. of Chicago, 1970.

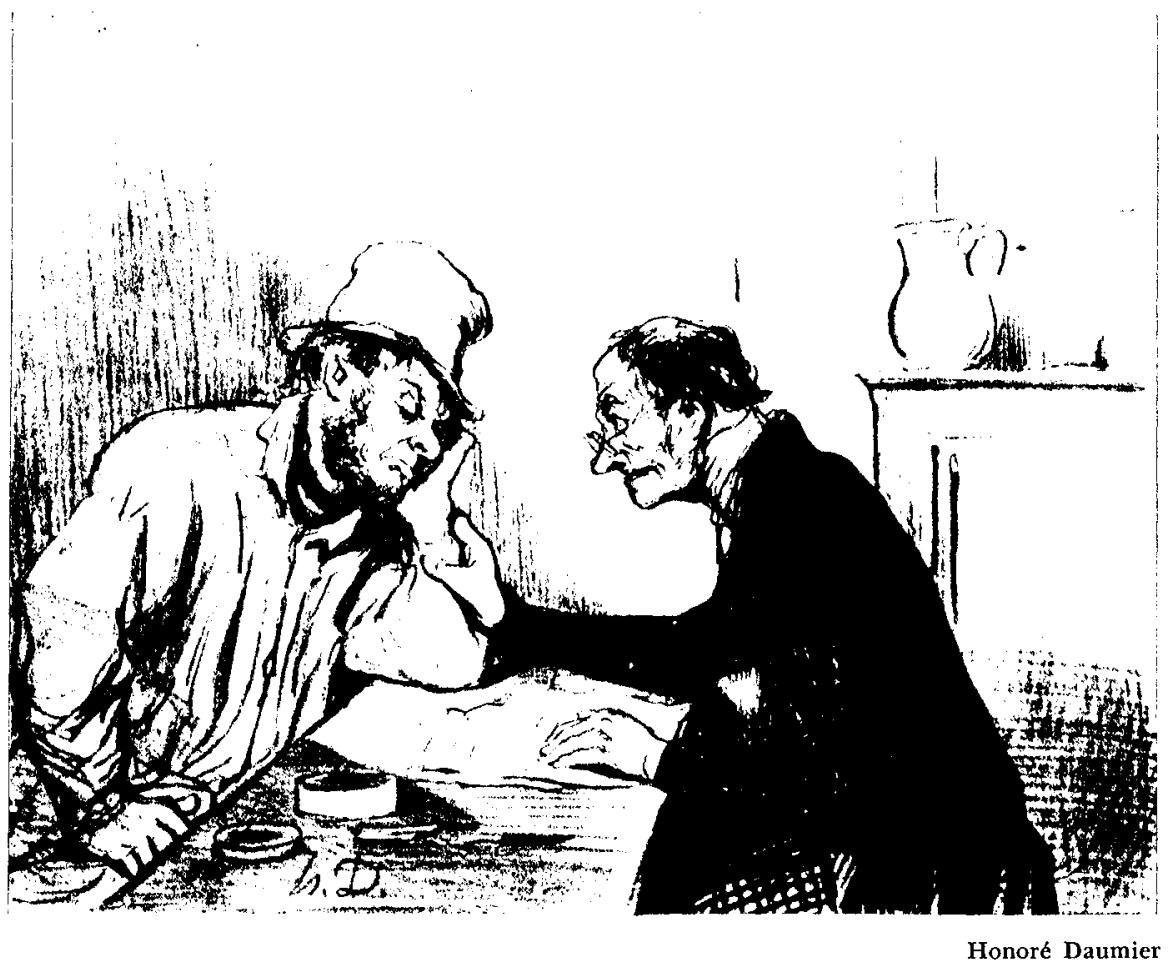

